\title{
RESOLUTION-RETRIEVING COMPENSATION OF SOURCE EFFECTS BY CORRELATIVE RECONSTRUCTION IN HIGH-RESOLUTION HOLOGRAPHY
}

\author{
G.W. STROKE, R. RESTRICK, A. FUNKHOUSER and D. BRUMM \\ The University of Michigan, Ann Arbor, Michigan
}

Received 26 July 1965

In this letter we show that the "spreading" effect due to the use of an extended source in the recording of a hologram does not result in an irretrievable resolution loss, but that the "loss" may be compensated and the resolution retrieved by illuminating the hologram, in the reconstruction, by the same source as that used in the recording (or by another suitable source), provided that the auto-correlation function of the function describing the "extended" source (or the crosscorrelation of the source functions) has itself a narrow central-peak width, of the order of the desired resolution. This is the case for suitably "structured" sources with a broad spatial-frequency representation, for example. We have verified the principle by using an arbitrarily selected "st ructured" source (fig. 1) in place of the usual point sources (recording and reconstruction) in a Fourier-transform holography arrangement [1-4]. With the use of (a) "lensless" Fourier-transform holography arrangements $[2,3]$ (having recordability gains, with spherical plates, exceeding $10^{3}$ compared to Fresneltransform holography), and (b) "structured source" holography arrangements, the two major limitations which appeared to stand in the way of extensions of high-resolution holography to the $\mathrm{X}$-ray domain now appear to have been largely cleared away.

Our method may at first sight appear to have some similarity with the arrangement independently considered by Denisyuk [5], who suggests using essentially "the same source" in the reconstruction, is in the recording. However, Denisyuk does not consider the resolution problem, nor indeed the special correlation requirements for the source, which are necessary for resolution-retrieving compensation in the reconstruction. We also do not consider here the use of "structured sources" as imaging or magnification aids in projection microscopy or holography [6].

For analysis of our method, we consider as a model the now usual one-dimensional Fouriertransform holography arrangements [1-4] which would normally use a "point" reference source $T_{\mathrm{S}}(\xi)=\delta(0)$ in the plane of the object $T_{\mathrm{o}}(\xi-a)$. However, in place of the point reference, we now use a spatially coherent, extended source, of complex amplitude $T_{\mathrm{s}}(\xi-a)$ and an object $T_{\mathrm{o}}(\xi)$, (see fig. 1), where $a$ is the usual off-set between the "center of gravity" of $T_{\mathrm{s}}$ and $T_{\mathrm{o}}$. The hologram intensity is $I(x)=\left[t_{\mathrm{o}} t_{\mathrm{o}}^{*}+t_{\mathrm{s}} t_{\mathrm{s}}^{*}\right]+$ $+t_{\mathrm{o}} t_{\mathrm{s}}{ }^{*} \exp [2 \pi \mathrm{i} a x]+t_{\mathrm{o}}{ }^{*} t_{\mathrm{s}} \exp [-2 \pi \mathrm{i} a x]$, where $t_{\mathrm{o}}$ and $t_{\mathbf{s}}$ are the Fourier transforms of $T_{\mathrm{o}}$ and $T_{\mathrm{s}}$, respectively. Under the now usual Fouriertransform reconstruction conditions [1], illumination of the hologram with a point source will produce an image, in which the two side-bands of interest will be respectively proportional to $\left[T_{\mathrm{o}} * T_{\mathrm{S}}\right]_{\xi-a}$ and to $\left[T_{\mathrm{o}} * * T_{\mathrm{s}}\right]_{\xi+a}$, where $*$

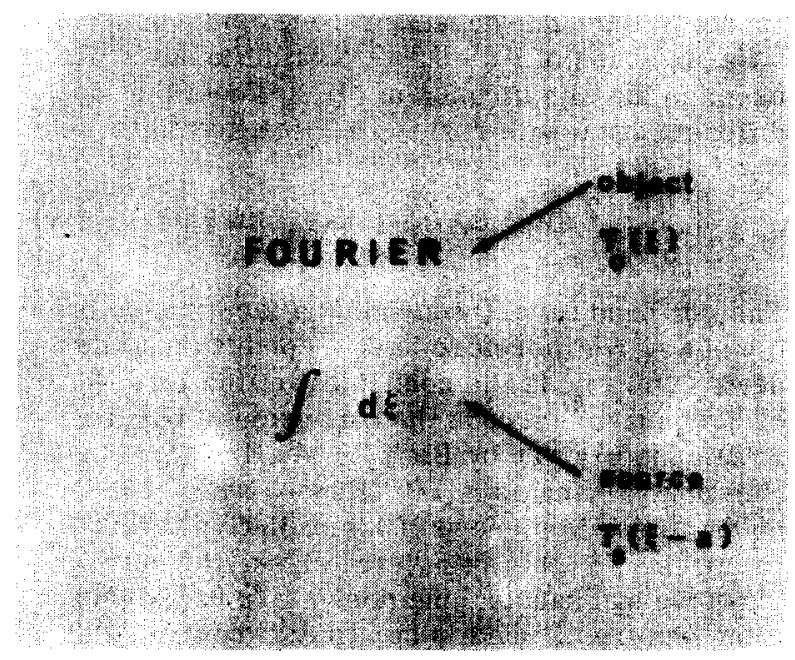

Fig. 1. Object "FOURIER" and source " $\int d \xi$ " (used in place of the usual "point" source), as used in recording of Fourier-transform hologram, from which the image of fig. 2 was reconstructed by Fourier-transformation. (Off-set $a=9 \mathrm{~mm}$, focal lengths of lenses used in Fourier-transformations $f=600 \mathrm{~mm}$ ). 


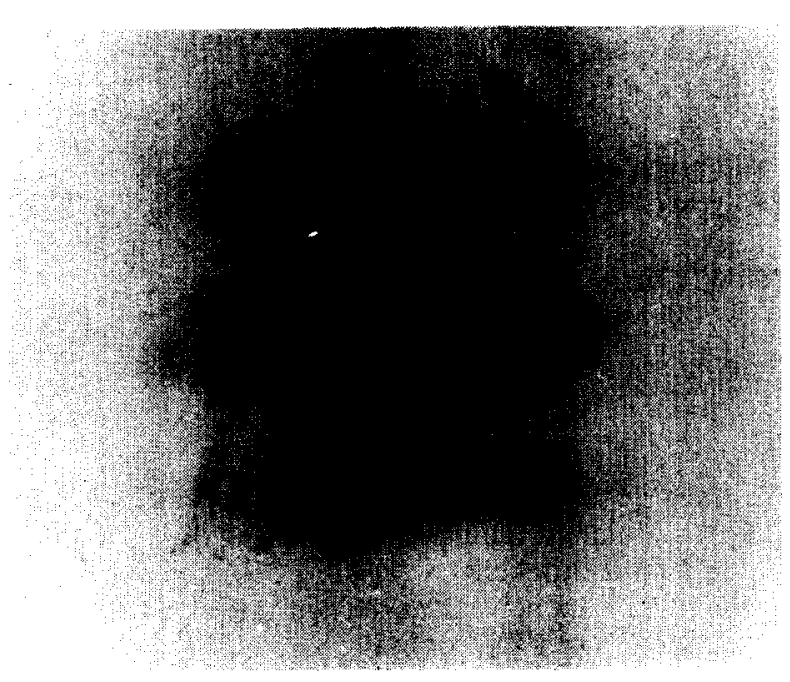

Fig. 2. Fourier-transform reconstruction of images obtained from hologram, recorded under conditions of fig. 1, by illuminating the hologram with a correlationcompensating source " $\int \mathrm{d} \xi$ " (the same as that used in the recording) used in place of the usual "point" source. Note that the upper side-band image of "FOURIER" is compensated by correlation of the source functions, while the lower side-band image is uncompensated, because of the convolution, rather than correlation, of the source functions (see text). (The entire holographic work was carried out in $6328 \mathrm{~A}$ coherent laser light, on Polaroid P/N film.)

indicates a correlation, and where the image coordinate is taken as $\xi$, under unit magnification. The images are seen to be "spread" out by correlation with the extended source. However, if we illuminate the hologram with a source $T_{\mathrm{s}}$, (which may or may not be equal to $T_{\mathrm{S}}$ ), having a transform $t_{\mathrm{s}}$, we find the complex amplitude transmission through the hologram, in the "upper" side band of interest, to be $t_{\mathrm{S}}\left[t_{0} t_{\mathrm{S}} * \times\right.$ $\times \exp (2 \pi \mathrm{i} a x)]=\left[t_{0} \exp (2 \pi \mathrm{i} a x)\right]\left[t_{\mathrm{S}}, t_{\mathrm{S}}{ }^{*}\right]$. The corresponding "upper" side-band image (see fig. 2), obtained by Fourier transformation, is $\left[T_{\mathrm{o}} \circledast\left(T_{\mathrm{S}}, * T_{\mathrm{S}}{ }^{*}\right)\right]_{\xi-a}$, which is equal to $\left[T_{\mathrm{o}}\right]_{\xi-a}$ if $\left[T_{\mathrm{s}}, * T_{\mathrm{s}}{ }^{*}\right]=\delta$, a delta function, (where we distinguish by $\circledast$ a convolution (rotate function in plane by $180^{\circ}$, shift and integrate overlapping areas), and by * a correlation (shift and integrate overlapping areas) [7]). If $T_{\mathrm{S}}$, is equal to $T_{\mathrm{S}}$ then $\left[T_{\mathrm{S}} * T_{\mathrm{S}}{ }^{*}\right]$ is the auto-correlation function of the source. For example, $\left[T_{\mathrm{S}} * T_{\mathrm{s}}{ }^{*}\right]$ will be a very narrow function if $T_{\mathrm{S}}$ can be represented by a very wide spectrum of spatial frequencies: in practice, such a representation would advantageously require an extended source field, and would therefore imply great luminosity. (An example is a Fresnel zone plate, or equivalent structure, possibly obtained by interference.) However, even in the case of the arbitrarily shaped source $\int \mathrm{d} \xi$, which we used in our experiments (fig. 1), a very clear "compensation" of the "spreading" was obtained (fig. 2). We note that the other (lower) side band of the hologram transforms (with $T_{\mathrm{S}^{\prime}}=T_{\mathrm{S}}$ ) into an image of the form $\left[T_{0}{ }^{*}\left(T_{\mathrm{S}} \circledast T_{\mathrm{S}}\right)\right]_{\xi+a}$. The convolution $\left(T_{\mathrm{S}} \circledast T_{\mathrm{S}}\right)$ will in general not be a delta function, except for sources having a two-fold rotation symmetry about the optical axis. The importance of the distinction between the desirable correlation of the source functions, $\left[T_{\mathrm{s}} * T_{\mathrm{s}}{ }^{*}\right]$ and the not always desirable convolution $\left(T_{\mathrm{S}} \circledast T_{\mathrm{S}}\right)$ appears in the "compensated" reconstruction of FOURIER in the upper side band (fig. 2), and the "uncompensated" reconstruction in the lower side band (fig. 2).

It follows from the preceeding analysis that the "twin image" in the original form of the Gabor microscope $[8,9]$ may be suppressed by the use of an extended source $T_{\mathrm{S}}$ having no twofold rotation symmetry. Applications to sourceeffect compensation in interferometry are equally clear, as are analogies with spectroscopic and other use of "matched" filtering $[10,11]$ and analogies with apodisation [12].

We wish to thank the National Science Foundation for its generous support of a part of this work.

\section{References}

1. G.W.Stroke and D.G. Falconer, Physics Letters 13 (1964) 306.

2. G.W.Stroke, Appl. Phys.Letters 6 (1965) 201.

3. G.W. Stroke, D. Brumm and A. Funkhouser, J. Opt. Soc. Am. 55 (1965) 10.

4. D. Gabor, G.W.Stroke, R. Restrick, A.Funkhouser and D. Brumm, Physics Letters 18 (1965)

5. Yu.N. Denisyuk, Dokl. Akad.Nauk SSSR 144 (1962) 1275; Soviet Phys.Dokl. Akad. Nauk SSSR 7 (1962) 543.

6. D.G. Falconer (unpublished work under the direction of G.W. Stroke) .

7. e.g. Y.W. Lee, Statigtical theory of communications (J.Wiley \& Sons, New York, 1960), p.46.

8. D. Gabor, Proc. Roy. Soc. (London) A197 (1949) 454.

9. D.Gabor, Proc. Roy. Soc. (London) B64 (1961) 449.

10. G.W. Stroke and D.G. Falconer, Physics Letters 15 (1965) 238.

11. e.g. A.Girard, Applied Optics 2 (1963) 79.

12. P. Jacquinot and B. Ruizen-Dossier, Progress in Optics, E. Wolf ed. (North-Holland Publishing Comp. Amsterdam, 1964) Vol.3, p. 136-146. 